\title{
RIGHT INVERSE SEMIGROUPS
}

\author{
S. MADHAVAN \\ (Received 22 November 1977; revised 26 April 1979) \\ Communicated by T. E. Hall
}

\begin{abstract}
In a recent paper of the author the well-known Vagner-Preston Theorem on inverse semigroups was generalized to include a wider class of semigroups, namely right normal right inverse semigroups. In an attempt to generalize the theorem to include all right inverse semigroups, the notion of $\mu-\mu_{i}$ transformations is introduced in the present paper. It is possible to construct a right inverse band $B_{M}(X)$ of $\mu-\mu_{i}$ transformations. From this a set $A_{M}(X)$ for which left and right units are in $B_{M}(X)$ and satisfying certain conditions is constructed. The semigroup $A_{M}(X)$ so constructed is a right inverse semigroup. Conversely every right inverse semigroup can be isomorphically embedded in a right inverse semigroup constructed in this way.
\end{abstract}

1980 Mathematics subject classification (Amer. Math. Soc.) : 20 M 20.

\section{Introduction}

A regular semigroup $S$ is called right inverse if for any idempotents $e, f$ of $S, e f e=f e$. Such semigroups have been studied by Ewing (1971), Venkatesan (1972), Bailes (1972) and Warne (1980). Right normal right inverse semigroups form a special class of right inverse semigroups and a faithful representation for these has been given by the author (1976). This generalizes the well-known Vagner-Preston Theorem ((1961), Theorem 1.20). An attempt is made in this paper to generalize these earlier theorems of the author to right inverse semigroups. For this, the notion of $\mu-\mu_{i}$ transformations is introduced. We can construct a right inverse band $B_{M}(X)$ of $\mu-\mu_{i}$ transformations; $B_{M}(X)$ is obviously a subsemigroup of the symmetric weakly inverse semigroup $T(X)$ of all partial transformations of $X$ (see Srinivasan (1968)). We define

$A_{M}(X)=\left\{\alpha: \alpha \in T(X)\right.$ and there exists an inverse $\alpha^{\prime}$ of $\alpha$ in $T(X)$ such that $\alpha^{\prime} \alpha$, $\alpha \alpha^{\prime} \in B_{M}(X)$ and for any $\left.\varepsilon \in B_{M}(X), \alpha \varepsilon \alpha^{\prime}, \alpha^{\prime} \varepsilon \alpha \in B_{M}(X)\right\}$. 
Then the semigroup $A_{M}(X)$ is a right inverse semigroup and it is called the symmetric right inverse semigroup on $(X, M)$.

\section{Basic concepts}

In general we follow the notation and terminology in Clifford and Preston(1961). For any $a \in S$, a regular semigroup, $V(a)$ denotes the set of inverses of the element $a$. A binary relation $\omega$ is called a quasi-ordering if it is reflexive and transitive. For any binary relation $\rho$, we denote by $U(\rho)$ and $U^{\prime}(\rho)$ its domain and range respectively.

In what follows $S$ is a right inverse semigroup.

LEMMA 2.1. For any $a, b \in S$, the following conditions are equivalent:

(i) There exists an idempotent $e$ in $S$ such that $a=b e$.

(ii) $a=b a^{\prime} a$ for any $a^{\prime} \in V(a)$.

ProOF. Assume (i). It is clear that there exists an inverse $a^{*}$ of $a$ such that $a^{*}=e b^{\prime}$, $b^{\prime}$ being an inverse of $b$. For any $a^{\prime} \in V(a), a^{*} a=a^{*} a a^{\prime} a a^{*} a=a^{\prime} a a^{*} a=a^{\prime} a$. Therefore, $b a^{\prime} a=b a^{*} a=b e\left(b^{\prime} b\right) e=b b^{\prime} b e=b e=a$. Thus we get (ii). Assume (ii). Then (i) is obvious.

We define $a \omega b$ on $S$ if (i) or (ii) and hence both the conditions in Lemma 2.1 are satisfied.

LEMMA 2.2. (Madhavan (1976)). The binary relation $\omega$ defines a compatible partial order on $S$.

It has been proved by Yamada (1967) that the binary relation $\mathscr{Y}$ on a generalized inverse semigroup $S$, defined as $(a, b) \in \mathscr{Y}$ if $a$ and $b$ have the same set of inverses, is the smallest inverse semigroup congruence on $S$. It has been subsequently shown by Hall (1969) that $\mathscr{Y}$ is the smallest inverse semigroup congruence on any orthodox semigroup.

LeMmA 2.3. (Madhavan (1976)). The following conditions are equivalent for any two elements $a, b$ in a right inverse semigroup $S$ :

(i) $(a, b) \in \mathscr{Y}$

(ii) $a \omega b, b \omega a$.

\section{The symmetric right inverse semigroup}

Let $X$ be a set and $M=\left\{\mu_{i}: i \in I\right\}$ be a commutative semigroup of equivalence relations whose domains are subsets of $X$. Assume that there is a maximum element 
$\mu$ in $M$. Define a $\mu-\mu_{i}$ transformation of $X$ as any map $\alpha$ from the subset $Y$ of $X$ onto a subset $Y \alpha$ of $X$ satisfying the following conditions :

M1. For any $x, y \in Y,(x, y) \in \mu_{i} \Leftrightarrow x \alpha=y \alpha$.

M2. For any $x, y \in Y,(x \alpha, y \alpha) \in \mu \Rightarrow(x, y) \in \mu$.

M3. If $x \in Y$ and $(x, y) \in \mu$, then $y \in Y$ and $(x \alpha, y \alpha) \in \mu$.

We note that the null map $\square$ vacuously satisfies these conditions and hence is a $\mu-\mu_{i}$ transformation.

To every $\mu-\mu_{i}$ transformation $\alpha$ we can associate a $\mu_{i}$ in $M$ and we denote $\mu_{i} \mid U(\alpha)=\alpha \circ \alpha^{-1}$ by $\mu(\alpha)$. A $\mu-\mu_{i}$ transformation $\alpha$ is called admissible if $\alpha^{2}=\alpha$ and for any $\mu-\mu_{j}$ transformation $\beta, \mu(\alpha \beta)=\mu(\alpha) \mu(\beta)$.

We denote the set of all admissible $\mu-\mu_{i}(i \in I)$ transformations by $B_{M}(X)$. We note that the null map $\square \in B_{M}(X)$.

LEMMA 3.1. A $\mu-\mu_{i}$ transformation $\alpha$ is an idempotent if and only if $\alpha \subseteq \mu_{i}$.

PROOF. This is true for the empty relation. Let $\alpha(\neq \square)$ be a $\mu-\mu_{i}$ transformation. If $\alpha^{2}=\alpha$, then $(x \alpha) \alpha=x \alpha$ whenever $x \alpha$ is defined, and this implies $(x, x \alpha) \in \mu_{i}$, whence $\alpha \subseteq \mu_{i}$. Conversely if $\alpha \subseteq \mu_{i}$ then for every $x$ for which $x \alpha$ is defined, we have $(x, x \alpha) \in \mu_{i}, \mu_{i} \subseteq \mu$ whence $x \in U(\alpha)$ by M3 and $x \alpha=(x \alpha) \alpha$ by M1. Thus $\alpha^{2}=\alpha$ as required. The lemma now follows.

LEMMA 3.2. Let $\alpha$ be an admissible $\mu-\mu_{i}$ transformation and $\beta$ a $\mu-\mu_{j}$ transformation. Then $\mu(\alpha \beta)=\mu_{i} \mu_{j} \mid U(\alpha \beta)$ and $\alpha \beta$ is a $\mu-\mu_{k}$ transformation, where $\mu_{k}=\mu_{i} \mu_{j}$

Proof. By hypothesis, $\mu(\alpha \beta)=\mu(\alpha) \mu(\beta)$ and clearly, $\mu(\alpha) \mu(\beta) \subseteq \mu_{i} \mu_{j} \mid U(\alpha \beta)$. Let $(x, y) \in \mu_{i} \mu_{j} \mid U(\alpha \beta)$. Then there exists $u$ such that $(x, u) \in \mu_{i},(u, y) \in \mu_{j}$. Since $x \in U(\alpha \beta)$ we get $x \in U(\alpha)$ and $x \alpha \in U(\beta)$. Clearly $x \alpha=u \alpha$. But $(u, u \alpha) \in \mu_{i}$ (Lemma 3.1). Since $x(\alpha \beta)$ is defined and $x \alpha=u \alpha$, we get that $u \alpha \beta$ is defined and hence $u \beta$ is defined. Consequently $u \beta=y \beta$. Thus $(x, u) \in \mu_{i} \mid U(\alpha)$ and $(u, y) \in \mu_{j} \mid U(\beta)$. It now follows that $\mu(\alpha \beta)=\mu(\alpha) \mu(\beta)=\mu_{i} \mu_{j} \mid U(\alpha \beta)$. Since $\mu_{i} \mu_{j} \in M$, denoting it by $\mu_{k}$ we find that we can associate $\mu_{\mathrm{k}} \in M$ with $\alpha \beta$. Clearly $(x, y) \in \mu_{\mathrm{k}} \Rightarrow x \alpha \beta=y \alpha \beta$ for any $x, y \in U(\alpha \beta)$. Also let $x \alpha \beta=y \alpha \beta$. Then $(x \alpha, y \alpha) \in \mu_{j}$. Since $\alpha^{2}=\alpha$ and $x \alpha$ is defined, $(x, x \alpha) \in \mu_{i}$ by Lemma 3.1. Thus $(x, x \alpha) \in \mu_{i}, \quad(x \alpha, y \alpha) \in \mu_{j} \Rightarrow(x, y \alpha) \in \mu_{i} \mu_{j}$. Similarly $(y, y \alpha) \in \mu_{i}$. Also $(x, y \alpha) \in \mu_{i} \mu_{j},(y, y \alpha) \in \mu_{i} \Rightarrow(x, y) \in \mu_{i} \mu_{j} \mu_{i}=\mu_{i} \mu_{j}=\mu_{k}$. Thus $\alpha \beta$ satisfies M1. Again $(x \alpha \beta, y \alpha \beta) \in \mu \Rightarrow \zeta(x \alpha, y \alpha) \in \mu \Rightarrow(x, y) \in \mu$. Thus $\mathrm{M} 2$ is satisfied. Let $x \in U(\alpha \beta), x \alpha \beta$ be defined and $(u, x) \in \mu$. Then $x \alpha$ is defined and therefore $(x \alpha, u \alpha) \in \mu$. This implies $(x \alpha \beta, u \alpha \beta) \in \mu$. Thus $\alpha \beta$ satisfies M3. Hence $\alpha \beta$ is a $\mu-\mu_{k}$ transformation.

Lemma 3.3. Let $\alpha, \beta \in B_{M}(X)$. Then $U(\alpha \beta)=U(\alpha) \cap U(\beta)$. 
ProOf. There exist $\mu_{i}, \mu_{j} \in M$ such that $\alpha \subseteq \mu_{i}$ and $\beta \subseteq \mu_{j}$. Then, let $x \in U(\alpha \beta)$. This implies that $x \in U(\alpha)$ and $x \alpha \in U(\beta)$. Clearly $(x, x \alpha) \in \mu$. Also $(x, x \alpha) \in \mu, x \alpha \in U(\beta)$ imply that $x \in U(\beta)$. We now get $x \in U(\alpha) \cap U(\beta)$. Thus we have shown that $U(\alpha \beta) \subseteq U(\alpha) \cap U(\beta)$. To prove the reverse inclusion, let $x \in U(\alpha) \cap U(\beta)$. Then $x \in U(\alpha),(x, x \alpha) \in \mu_{i} \subseteq \mu$ and $x \alpha \in U(\beta)$. These imply that $x \in U(\alpha)$ and $x \alpha \in U(\beta)$ which in turn imply that $x \in U(\alpha \beta)$. Thus we get $U(\alpha) \cap U(\beta) \subseteq U(\alpha \beta)$. The lemma now follows.

LEMMA 3.4. If $\alpha, \beta \in B_{M}(X)$, then $\mu(\alpha) \mu(\beta)=\mu(\beta) \mu(\alpha)$.

PROOF. There exist $\mu_{i}, \mu_{j} \in M$ such that $\alpha \subseteq \mu_{i}$ and $\beta \subseteq \mu_{j}$. Let $(x, y) \in \mu(\alpha) \mu(\beta)$. This implies that there exists $u \in X$ such that $(x, u) \in \mu_{i}, x \in U(\alpha),(u, y) \in \mu_{j}$ and $u \in U(\beta)$ for some $u \in X$. Thus we get $(x, y) \in \mu_{i} \mu_{j}$. Also $u \in U(\beta),(x, u) \in \mu_{i} \subseteq \mu$ imply that $x \in U(\beta)$. Again $x \in U(\alpha), x \in U(\beta)$ imply that $x \in U(\alpha) \cap U(\beta)$. Thus we get $(x, y) \in \mu_{i} \mu_{j}$ and $x \in U(\alpha) \cap U(\beta)=U(\alpha \beta)=U(\beta \alpha)$. Since $\mu_{i} \mu_{j}=\mu_{j} \mu_{i}$ it follows that $(x, y) \in \mu(\beta) \mu(\alpha)$, whence we get $\mu(\alpha) \mu(\beta) \subseteq \mu(\beta) \mu(\alpha)$. The reverse inclusion can be similarly proved. The lemma now follows.

THEOREM 3.1. $B_{M}(X)$ is a right inverse band.

Proof. Let $\alpha, \beta \in B_{M}(X)$. It is clear from Lemma 3.1 that $\alpha \beta$ satisfies M1, M2 and M3. We shall show that $\alpha \beta \alpha=\beta \alpha$. For this, let $x \in U(\alpha \beta \alpha)$. Then there exists $v \in U(\beta)$ such that $(x \alpha, v) \in \mu(\beta)$ and $x \alpha \beta=v \beta$. Therefore $x \alpha \beta \alpha=v \beta \alpha$. We note that for any $\xi, \eta \in B_{M}(X), \quad \mu(\xi \eta)=\mu(\eta \xi) \quad$ in view of Lemma 3.4 and therefore $x \xi \eta=y \xi \eta \Leftrightarrow x \eta \xi=y \eta \xi$. Thus $(x \alpha) \beta \alpha=v \beta \alpha \Rightarrow(x \alpha) \alpha \beta=v \alpha \beta \Rightarrow x \beta \alpha=v \beta \alpha=x \alpha \beta \alpha$. Therefore $\alpha \beta \alpha \subseteq \beta \alpha$. Now let $x \in U(\alpha \beta)$. We note that $x \alpha \beta$ is defined if and only if $x \beta \alpha$ is defined. When $x \in U(\alpha \beta)$, there exists $v \in X$ such that $(x \alpha, v) \in \mu(\beta)$. Then $x \alpha \beta=v \beta$. Now,

$$
x \alpha \beta=v \beta \Rightarrow x \alpha \beta \alpha=v \beta \alpha \Rightarrow(x \alpha) \alpha \beta=v \alpha \beta \Rightarrow(x \alpha) \beta=v \alpha \beta \Rightarrow x \beta \alpha=v \beta \alpha=x \alpha \beta \alpha .
$$

Therefore $\beta \alpha \subseteq \alpha \beta \alpha$. Combining this with the earlier result we get $\alpha \beta \alpha=\beta \alpha$.

It remains to show that $\alpha \beta$ is admissible. Clearly $\alpha \beta$ is idempotent. Let $\gamma$ be a $\mu-\mu_{i}$ transformation. Then by Lemma 3.1, $\beta \gamma$ satsfies M1, M2 and M3 and hence is a $\mu-\mu_{j}$ transformation for some $j \in I$. Then

$$
\mu(\alpha \beta \gamma)=\mu(\alpha) \mu(\beta \gamma)=\mu(\alpha) \mu(\beta) \mu(\gamma)=\mu(\alpha \beta) \mu(\gamma) .
$$

The theorem now follows.

Let $T(X)$ denote the (symmetric weakly inverse) semigroup of all partial transformations of $X$. (See Srinivasan (1968).) 
THEOREM 3.2. Let

$$
\begin{gathered}
A_{M}(X)=\left\{\alpha \in T(X): \text { there exists an inverse } \alpha^{\prime} \text { of } \alpha \text { in } T(X) \text { such that } \alpha^{\prime} \alpha, \alpha \alpha^{\prime} \in B_{M}(X)\right. \\
\text { and for any } \left.\varepsilon \in B_{M}(X), \alpha^{\prime} \varepsilon \alpha, \alpha \varepsilon \alpha^{\prime} \in B_{M}(X)\right\} .
\end{gathered}
$$

Then $A_{M}(X)$ is a right inverse semigroup.

Proof. We shall first show that $A_{M}(X)$ is a semigroup. Let $\alpha, \beta \in B_{M}(X)$. Then there exist inverses $\alpha^{\prime}$ of $\alpha, \beta^{\prime}$ of $\beta$ such that $\alpha^{\prime} \alpha, \alpha \alpha^{\prime}, \beta^{\prime} \beta, \beta \beta^{\prime} \in B_{M}(X)$ and satisfying the conditions mentioned above. We note that

$$
\alpha \beta \beta^{\prime} \alpha^{\prime} \alpha \beta=\alpha \beta \beta^{\prime} \alpha^{\prime} \alpha \beta \beta^{\prime} \beta=\alpha \alpha^{\prime} \alpha \beta \beta^{\prime} \beta=\alpha \beta,
$$

since $\alpha^{\prime} \alpha, \beta \beta^{\prime} \in B_{M}(X)$. Also, similarly

$$
\beta^{\prime} \alpha^{\prime} \alpha \beta \beta^{\prime} \alpha^{\prime}=\beta^{\prime} \alpha^{\prime} \alpha \beta \beta^{\prime} \alpha^{\prime} \alpha \alpha^{\prime}=\beta^{\prime} \beta \beta^{\prime} \alpha^{\prime} \alpha \alpha^{\prime}=\beta^{\prime} \alpha^{\prime} .
$$

Therefore there exists an inverse $\beta^{\prime} \alpha^{\prime} \in T(X)$ and $\alpha \beta \beta^{\prime} \alpha^{\prime}, \beta^{\prime} \alpha^{\prime} \alpha \beta \in B_{M}(X)$ by the definition of $A_{M}(X)$. Also for any $\varepsilon \in B_{M}(X), \alpha \beta \varepsilon \beta^{\prime} \alpha^{\prime}, \beta^{\prime} \alpha^{\prime} \varepsilon \alpha \beta \in B_{M}(X)$. Thus $\alpha \beta \in A_{M}(X)$. It follows that $A_{M}(X)$ is a semigroup. We shall show that $A_{M}(X)$ is right inverse. For this, let $\alpha=\alpha^{2} \in A_{M}(X)$. Then there exists an inverse $\alpha^{\prime}$ of $\alpha$ in $T(X)$ such that $\alpha \alpha^{\prime}, \alpha^{\prime} \alpha \in B_{M}(X)$. Since $B_{M}(X)$ is a semigroup we get $\alpha^{\prime}=\alpha^{\prime} \alpha \alpha^{\prime}=\left(\alpha^{\prime} \alpha\right)\left(\alpha \alpha^{\prime}\right) \in B_{M}(X)$. Thus $\alpha^{\prime}$ is an idempotent and $\alpha=\alpha \alpha^{\prime} \alpha=\left(\alpha \alpha^{\prime}\right)\left(\alpha^{\prime} \alpha\right) \in B_{M}(X)$. Therefore if $\alpha=\alpha^{2} \in A_{M}(X)$, then $\alpha \in B_{M}(X)$. Thus $B_{M}(X)$ is the subsemigroup of idempotents of $A_{M}(X)$. It remains to show that $A_{M}(X)$ is regular. For this, let $\alpha \in A_{M}(X)$. By definition, there exists $\alpha^{\prime} \in T(X)$ such that $\alpha \alpha^{\prime} \alpha=\alpha, \alpha^{\prime} \alpha \alpha^{\prime}=\alpha^{\prime}$ and $\alpha \alpha^{\prime}, \alpha^{\prime} \alpha \in B_{M}(X)$. Also for any $\varepsilon \in B_{M}(X), \alpha^{\prime} \varepsilon \alpha, \alpha \varepsilon \alpha^{\prime} \in B_{M}(X)$. Consequently $\alpha^{\prime} \in A_{M}(X)$, whence we get $A_{M}(X)$ is regular. This together with the earlier assertion, shows that $A_{M}(X)$ is a right inverse semigroup.

\section{The embedding theorem}

Before proving the final embedding theorem we need the following lemma proved by Madhavan (1976).

LeMmA 4.1. If $S$ is an orthodox semigroup, $a \in S$ and $a^{\prime}, a^{*} \in V(a)$, then $\left(S a^{\prime}\right) \mathscr{Y}=\left(S a^{*}\right) \mathscr{Y}, \mathscr{Y}$ being the Yamada-Hall congruence on $S$.

Let $S$ be a right inverse semigroup. For all $e=e^{2} \in S$, define

$$
\mathscr{Y}_{e}=\{(x, y) \in(\mathrm{Se}) \mathscr{Y} \times(\mathrm{Se}) \mathscr{Y}: x e=y e\} .
$$

Clearly $\mathscr{Y}_{e} \subseteq \mathscr{Y}$. We have then :

LeMma 4.2. Let $e=e^{2}, f=f^{2} \in S$. Then $\mathscr{Y}_{e} \mathscr{Y}_{f}=\mathscr{Y}_{f} \mathscr{Y}_{e}=\mathscr{Y}_{e f}$. 
ProOf. Let $(x, y) \in \mathscr{Y}_{e} \mathscr{Y}_{f}$. There exists $u \in S$ such that $(x, u) \in \mathscr{Y}_{e}$ and $(u, y) \in \mathscr{Y}_{f}$. Now $x e=u e \Rightarrow x e f=u e f=u f e f$. Also $u f e f=y f e f=y e f$. Thus it follows that $x e f=y e f$. Clearly $x \in(S e) \mathscr{Y}$ and $y \in(S f) \mathscr{Y}$. There exists $k \in S$ such that $(x, k e) \in \mathscr{Y}$ and this implies $(x e, k e) \in \mathscr{Y}$. But $(x e, k e) \in \mathscr{Y},(x, k e) \in \mathscr{Y} \Rightarrow(x, x e) \in \mathscr{Y}$. Since $x e=u e$ it follows that $(x, u e) \in \mathscr{Y}$. Also $(x, u e) \in \mathscr{Y}, \quad(x, u) \in \mathscr{Y}_{e} \Rightarrow(u, u e) \in \mathscr{Y}$; $(u, u e) \in \mathscr{Y} \Rightarrow(u f, u e f) \in \mathscr{Y} ;(u, u f) \in \mathscr{Y},(u f, u e f) \in \mathscr{Y} \Rightarrow(u, u e f) \in \mathscr{Y} \Rightarrow($ ue, uef $) \in \mathscr{Y}$ since $(u, u e) \in \mathscr{Y}$. Again $(x, u e) \in \mathscr{Y},(u e, u e f) \in \mathscr{Y} \Rightarrow(x, u e f) \in \mathscr{Y}$. Thus $x \in($ Sef $) \mathscr{Y}$. Also $(y, y f) \in \mathscr{Y}$. But $y f=u f$ and thus we get $(y, u f) \in \mathscr{Y}$. Also

$$
(u, u f) \in \mathscr{Y} \Rightarrow(u e, u f e) \in \mathscr{Y} \Rightarrow(u, u f e) \in \mathscr{Y} \Rightarrow(u f, u f e f) \in \mathscr{Y} \Rightarrow(u f, u e f) \in \mathscr{Y} \Rightarrow(y, u f) \in \mathscr{Y}
$$

and $(u f, u e f) \in \mathscr{Y} \Rightarrow(y, u e f) \in \mathscr{Y}$. Thus $y \in($ Sef $) \mathscr{Y}$. It now follows that $(x, y) \in \mathscr{Y}_{\text {ef }}$ whence we get $\mathscr{Y}_{e} \mathscr{Y}_{f} \subseteq \mathscr{Y}_{e f}$. To prove the reverse inclusion, let $(x, y) \in \mathscr{Y}_{\text {ef }}$. There exists $u \in S$ such that $(x, u e f) \in \mathscr{Y}$. Then $(x e f, u e f) \in \mathscr{Y}$ and this implies

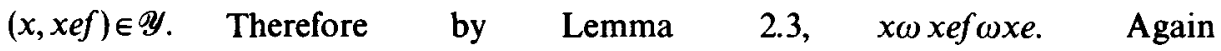
$(x, x e f) \in \mathscr{Y} \Rightarrow(x e, x e f e) \in \mathscr{Y} \Rightarrow(x e, x f e) \in \mathscr{Y}$. Therefore $x e \omega x f e \omega x$. Then $(x, x e) \in \mathscr{Y}$, whence we get $x \in(\mathrm{Se}) \mathscr{Y}$. Also $x e \in(\mathrm{Se}) \mathscr{Y}$ and $x e f \in(S e f) \mathscr{Y}$. As proved earlier $(x, x e f) \in \mathscr{Y}$ and this together with $(x, x e) \in \mathscr{Y}$ implies $(x e, x e f) \in \mathscr{Y}$. Now $(x e, x e f) \in \mathscr{Y} \Rightarrow x e \in(S e f) \mathscr{Y} \subseteq(S f) \mathscr{Y}$. Clearly, $x e f \in(S e f) \mathscr{Y} \subseteq(S f) \mathscr{Y}$. It now follows that $(x, x e) \in \mathscr{Y}_{e}$ and $(x e, x e f) \in \mathscr{Y}_{f}$. We get $\mathscr{Y}_{e f} \subseteq \mathscr{Y}_{e} \mathscr{Y}_{f}$. Combining with the previous result, we get $\mathscr{Y}_{e} \mathscr{Y}_{f}=\mathscr{Y}_{e f}$.

To prove that $\mathscr{Y}_{e} \mathscr{Y}_{f}=\mathscr{Y}_{f} \mathscr{Y}_{e}$, we first note that for any $x, y \in S$,

$$
x e f=y e f \Rightarrow x e f e=y e f e \Rightarrow x f e=y f e \Rightarrow x f e f=y f e f \Rightarrow x e f=y e f .
$$

Also let $x \in(\operatorname{Sef}) \mathscr{Y}$. Then there exists $u \in S$ such that $(x, u e f) \in \mathscr{Y}$. Now $(x, u e f) \in \mathscr{Y} \Rightarrow x \omega x e f \omega x f e \omega x$. Thus $(x, x f e) \in \mathscr{Y}$. This implies that $x \in(S f e) \mathscr{Y}$. Thus we get $(S e f) \mathscr{Y} \subseteq(S f e) \mathscr{Y}$. The reverse inclusion can be similarly proved whence we get $(S e f) \mathscr{Y}=(S f e) \mathscr{Y}$. Thus we have shown that $\mathscr{Y}_{e f}=\mathscr{Y}_{f e}$, whence the lemma follows.

THEOREM 4.1. Let $S$ be a right inverse semigroup. Then for every $a \in S$ define $\rho_{a}:\left(S a^{\prime}\right) \mathscr{Y} \rightarrow$ Saby $x \rho_{\alpha}=x a$. Then $a \rightarrow \rho_{a}(a \in S)$ is a monomorphism from $S$ into $T(S)$.

Proof. Let $a \in S$ and $a^{\prime} \in V(a)$. We shall show that $a \rightarrow \rho_{a}$ defines a monomorphism. For this it is enough to show that $U\left(\rho_{a} \rho_{b}\right)=U\left(\rho_{a b}\right)$. Let $x \in U\left(\rho_{a} \rho_{b}\right)$. For any $a^{\prime} \in V(a),\left(x, x a a^{\prime}\right) \in \mathscr{Y}$ and there exists $u \in\left(S b b^{\prime}\right) \mathscr{Y}$ for any $b^{\prime} \in V(b)$, such that $(x a, u) \in \mathscr{Y}$. Obviously, $\left(u, u b b^{\prime}\right) \in \mathscr{Y}$ and therefore, $\left(x a, u b b^{\prime}\right) \in \mathscr{Y}$. Now $\left(x a, u b b^{\prime}\right) \in \mathscr{Y} \Rightarrow\left(x a a^{\prime}, u b b^{\prime} a^{\prime}\right) \in \mathscr{Y}$ and $\left(x, u b b^{\prime} a^{\prime}\right) \in \mathscr{Y} \Rightarrow x \in U\left(\rho_{a b}\right)$. Let $x \in U\left(\rho_{a b}\right)$. Then for any $b^{\prime} \in V(b)$, and any $a^{\prime} \in V(a)$, $\left(x, x a b b^{\prime} a^{\prime}\right) \in \mathscr{Y}$. Clearly, $x a b b^{\prime} a^{\prime} \in S a^{\prime}$ and therefore $x \in\left(S a^{\prime}\right) \mathscr{Y}$. Now

$\left(x, x a b b^{\prime} a^{\prime}\right) \in \mathscr{Y} \Rightarrow\left(x a, x a b b^{\prime} a^{\prime} a\right) \in \mathscr{Y} \Rightarrow x a \omega x a b b^{\prime} a^{x} a \omega x a b b^{\prime} \omega x a$. 
Thus $\left(x a, x a b b^{\prime}\right) \in \mathscr{Y}$, whence we get $x a \in\left(S b^{\prime}\right) \mathscr{Y}$. Therefore, $x \in\left(S a \cap\left(S b^{\prime}\right) \mathscr{Y}\right) \rho_{a}^{-1}$. It now follows that $\rho_{a} \rho_{b}=\rho_{a b}$. To show that for any $a, b \in S, \rho_{a}=\rho_{b} \Rightarrow a=b$, let $\rho_{a}=\rho_{b}$. Then $S a=S b$ and therefore $a^{\prime} a=b^{\prime} b$ for any $a^{\prime} \in V(a)$ and any $b^{\prime}=V(b)$, $b^{\prime} \in\left(S b^{\prime}\right) \mathscr{Y}=\left(S a^{\prime}\right) \mathscr{Y}$. Thus $a^{\prime} b=a^{\prime} a=b^{\prime} b=b^{\prime} a$. We get $a\left(b^{\prime} a\right) b^{\prime}=a b^{\prime} b b^{\prime}=a b^{\prime}$. Thus $a b^{\prime}$ is idempotent. Then

$$
a b^{\prime}=a b^{\prime} b b^{\prime} b b^{\prime}=a b^{\prime} b b^{\prime} a b^{\prime}=b b^{\prime} b b^{\prime}=b b^{\prime}
$$

and

$$
a=a a^{\prime} a=a b^{\prime} a=b b^{\prime} a=b b^{\prime} b=b .
$$

We can define $M=\{\mathscr{Y}\} \cup\left\{\mathscr{Y}_{e}: e=e^{2} \in S\right\}$. Clearly $M$ is a commutative semigroup of equivalence relations whose domains are subsets of $S$; $\mathscr{Y}$ is the maximum element. We can define a $\mathscr{Y}-\mathscr{Y}_{e}$ transformation satisfying M1, M2 and M3. To every such $\mathscr{Y}-\mathscr{Y}_{e}$ transformation we can associate an equivalence relation $\mathscr{Y}_{e}$.

We denote the set $\left\{x \rho_{e}: x \in U\left(\rho_{e}\right)\right\}$ by $S \rho_{e}$.

LEMMA 4.3. Let $e=e^{2} \in S, f=f^{2} \in S$ and $\alpha$ be any $\mathscr{Y}-\mathscr{Y}_{f}$ transformation and $\alpha \subseteq \rho_{f}$. Then $\alpha \rho_{e} \alpha=\rho_{e} \alpha$.

Proof. Let $x \alpha \rho_{e} \alpha$ be defined. Then $x \alpha \rho_{e} \alpha=x \rho_{f} \rho_{e} \rho_{f}=x \rho_{e} \rho_{f}$. Since $\left(x, x \rho_{e}\right) \in \mathscr{Y}$ and $x \alpha$ is defined, $x e \alpha$ is defined and therefore $x \rho_{e} \rho_{f}=x \rho_{e} \alpha$. Thus $x \alpha \rho_{e} \alpha=x \rho_{e} \alpha$. This implies $\alpha \rho_{e} \alpha \subseteq \rho_{e} \alpha$. Conversely, let $x \rho_{e} \alpha$ be defined. Then

$$
x \rho_{e} \alpha=x \rho_{e} \rho_{f}=x \rho_{f} \rho_{e} \rho_{f} .
$$

Since $\left(x, x \rho_{e}\right) \in \rho_{e} \subseteq \mathscr{Y}$ and $\left(x \rho_{e}\right) \alpha$ is defined we have that $x \alpha$ is defined. Also $\left(x, x \rho_{f} \rho_{e}\right) \in \mathscr{Y}$ and this implies, since $x \alpha$ is defined, that $\left(x \rho_{f} \rho_{e}\right) \alpha$ is defined. Thus $x \rho_{e} \alpha=x \rho_{f} \rho_{e} \rho_{f}=(x \alpha) \rho_{e} \alpha$. Therefore $\rho_{e} \alpha \subseteq \alpha \rho_{e} \alpha$ and combining this with the earlier result, we get $\alpha \rho_{e} \alpha=\rho_{e} \alpha$. The lemma now follows. If $e=e^{2} \in S, \alpha \subseteq \rho_{e}$ and $\alpha$ is a $\mathscr{Y}-\mathscr{Y}_{e}$ transformation, then $\alpha \subseteq \rho_{e} \subseteq \mathscr{Y}\left(\rho_{e}\right)$. Thus $\alpha^{2}=\alpha$, by Lemma 3.1.

LeMma 4.4. Let $e=e^{2}, f=f^{2} \in S$ and $\beta$ a $\mathscr{Y}-\mathscr{Y}_{f}$ transformation such that $\beta \subseteq \rho_{f}$. Then $(x, y) \in \mathscr{Y}\left(\rho_{e} \beta\right)$ if and only if $(x, y) \in \mathscr{Y}\left(\rho_{e} \rho_{f}\right)$ and $(y, y) \in \mathscr{Y}(\beta)$.

Proof. Let $(x, y) \in \mathscr{Y}\left(\rho_{e} \beta\right)$. Then clearly $(x, y) \in \mathscr{Y}\left(\rho_{e} \rho_{f}\right)$. Also $\left(x, x \rho_{e} \beta\right) \in \mathscr{Y}$. Now $\left(x, x \rho_{e} \beta\right) \in \mathscr{Y} \Rightarrow x \in\left(S \rho_{e} \beta\right) \mathscr{Y} \subseteq(S \beta) \mathscr{Y}$.

Thus $(y, y) \in \mathscr{Y}(\beta)$. To prove the converse, we note that $(x, y) \in \mathscr{Y}\left(\rho_{e} \rho_{f}\right)$, $(y, y) \in \mathscr{Y}(\beta) \Rightarrow x, y \in U\left[\mathscr{Y}\left(\rho_{e} \rho_{f}\right)\right], x \rho_{e} \rho_{f}=y \rho_{e} \rho_{f}, y \rho_{f}=y \beta$. These imply that $x, y \in U\left[\mathscr{Y}\left(\rho_{e} \rho_{f}\right)\right], x \rho_{f} \rho_{e}=y \rho_{f} \rho_{e}, y \rho_{f}=y \beta$. These again imply

$$
x \rho_{e} \rho_{f}=x \rho_{f} \rho_{e} \rho_{f}=y \rho_{f} \rho_{e} \rho_{f}=y \rho_{e} \rho_{f}
$$


and

$$
y \rho_{f} \rho_{e} \beta=y \beta \rho_{e} \beta=y \rho_{e} \beta .
$$

We note that $y \beta$ is defined and $\left(y, y \beta_{e}\right) \in \mathscr{Y}$. Therefore $y \beta \rho_{e} \beta$ is defined. Hence

$$
y \beta \rho_{e} \beta=y \rho_{e} \beta=y \rho_{e} \rho_{f}=x \rho_{e} \rho_{f}
$$

But

$$
x \rho_{e} \rho_{f}=y \rho_{e} \rho_{f} \Rightarrow\left(x \rho_{e}, y \rho_{e}\right) \in \mathscr{Y}\left(\rho_{f}\right) \subseteq \mathscr{Y} .
$$

Since $y \rho_{e} \beta$ is defined, it now follows that $x \rho_{e} \beta=x \rho_{e} \rho_{f}=y \rho_{e} \beta$. Thus we get $x, y \in U\left[\mathscr{Y}\left(\rho_{e} \rho_{f}\right)\right]$ and $x \rho_{e} \beta=y \rho_{e} \beta$. Since $x \rho_{e} \beta$ is defined it follows that $x \in U\left[\mathscr{Y}\left(\rho_{e} \beta\right)\right]$ and similarly for $y$. Thus we get $x, y \in U\left[\mathscr{Y}\left(\rho_{e} \beta\right)\right]$ and $x \rho_{e} \beta=y \rho_{e} \beta$. It is now immediate that $(x, y) \in \mathscr{Y}\left(\rho_{e} \beta\right)$.

LEMMA 4.5. Let $e=e^{2} \in S$ and let $\alpha$ be a $\mathscr{Y}-\mathscr{Y}_{e}$ transformation such that $\alpha \subseteq \rho_{e}$. Then $(x, y) \in \mathscr{Y}(\alpha)$ if and only if $(x, y) \in \mathscr{Y}\left(\rho_{e}\right)$ and $(y, y) \in \mathscr{Y}(\alpha)$.

PROOF. Now, $\quad(x, y) \in \mathscr{Y}(\alpha) \Rightarrow(x, y) \in \mathscr{Y}\left(\rho_{e}\right), \quad(y, y) \in \mathscr{Y}(\alpha) \Rightarrow x, y \in U\left[\mathscr{Y}\left(\rho_{e}\right)\right]$, $x \rho_{e}=y \rho_{e}=y \alpha \Rightarrow x, y \in U\left[\mathscr{Y}\left(\rho_{e}\right)\right], x \alpha=y \alpha$ since $(x, y) \in \mathscr{Y}\left(\rho_{e}\right) \subseteq \mathscr{Y}$ and hence $x \alpha$ is defined whence $x \rho_{e}=x \alpha$. But $x \in U\left[\mathscr{Y}\left(\rho_{e}\right)\right]$ and $x \alpha$ is defined implies $x \in U[\mathscr{Y}(\alpha)]$ and similarly for $y$. This proves the lemma.

LEMMA 4.6. Let $e=e^{2}, f=f^{2} \in S$ and let $\beta$ be $a \mathscr{Y}-\mathscr{Y}_{f}$ transformation such that $\beta \subseteq \rho_{f}$. Then $\mathscr{Y}\left(\rho_{e} \beta\right)=\mathscr{Y}\left(\rho_{e}\right) \mathscr{Y}(\beta)$.

ProOf. Now,

$$
\begin{gathered}
(x, y) \in \mathscr{Y}\left(\rho_{e} \beta\right) \leftrightarrow(x, y) \in \mathscr{Y}\left(\rho_{e} \rho_{f}\right), \\
(y, y) \in \mathscr{Y}(\beta) \Leftrightarrow(x, u) \in \mathscr{Y}\left(\rho_{e}\right) \text { and }(u, y) \in \mathscr{Y}\left(\rho_{f}\right)
\end{gathered}
$$

for some $u \in S,(y, y) \in \mathscr{Y}(\beta) \Leftrightarrow(x, u) \in \mathscr{Y}\left(\rho_{e}\right),(u, y) \in \mathscr{Y}(\beta) \Leftrightarrow(x, y) \in \mathscr{Y}\left(\rho_{e} \beta\right)$. The lemma now follows.

Now let $e=e^{2} \in S$ so that $\rho_{e}$ is a $\mathscr{Y}-\mathscr{Y}_{e}$ transformation. Let $\beta$ be a $\mathscr{Y}-\mathscr{Y}_{f}$ transformation. Then there exists $\beta^{\prime}$, an inverse of $\beta$ such that $\beta \beta^{\prime} \subseteq \rho_{f}$. Clearly, $\mathscr{Y}\left(\beta \beta^{\prime}\right)=\mathscr{Y}(\beta)$. It is easy to verify that $\beta \beta^{\prime}$ is a $\mathscr{Y}-\mathscr{Y}_{f}$ transformation.

LEMMA 4.7. Let $e=e^{2} \in S$. Then $\rho_{e}$ is an admissible $\mathscr{Y}-\mathscr{Y}_{e}$ transformation.

Proof. Clearly $\rho_{e}^{2}=\rho_{e}$. Let $\beta$ be a $\mathscr{Y}-\mathscr{Y}_{f}$ transformation. Then by Lemma 4.5, and the above observation we get $\mathscr{Y}\left(\rho_{e} \beta\right)=\mathscr{Y}\left(\rho_{e}\right) \mathscr{Y}(\beta)$. The lemma now follows. 
THEOREM 4.2. Let $S$ be a right inverse semigroup and

$$
M=\{\mathscr{Y}\} \cup\left\{\mathscr{Y}_{e}: e=e^{2} \in S\right\} .
$$

Then $S$ can be isomorphically embedded in the symmetric right inverse semigroup $T(S, M)$ on $(S, M)$.

Proof. We have seen that $\left\{\rho_{e}: e=e^{2} \in S\right\}$ forms a right inverse band $E$ of admissible $\mathscr{Y}-\mathscr{Y}_{e}$ transformations on $S$. Define

$A=\left\{\alpha: \alpha \in T(S), \alpha \alpha^{\prime}, \alpha^{\prime} \alpha \in E\right.$ for some inverse $\alpha^{\prime}$ of $\alpha$, and for every $\left.\varepsilon \in E, \alpha \varepsilon \alpha^{\prime}, \alpha^{\prime} \varepsilon \alpha \in E\right\}$.

Then clearly $S$ is embedded isomorphically in $A=T(S, M)$ by virtue of Theorem 4.1.

ADDED IN PROOF : In conclusion the author expresses his sincere thanks to Dr T. E. Hall for many valuable suggestions that have led to considerable improvement of this paper.

\section{References}

G. L. Bailes, (1972), Right inverse semigroups, Doctoral dissertation, Clemson University.

A. H. Clifford and G. B. Preston (1961), The algebraic theory of semigroups, Vol. I (Maths. Surveys No. 7, Amer. Math. Soc., Providence, R.I.).

E. W. Ewing (1971), Contribution to the study of regular semigroups, Doctoral dissertation, University of Kentucky.

T. E. Hall (1969), 'On regular semigroups whose idempotents form a subsemigroup, Bull. Australian Math. Soc. 1, 195-208.

S. Madhavan (1976), 'On right normal right inverse semigroups', Semigroup Forum 12, 333-339.

B. R. Srinivasan (1968), 'Weakly inverse semigroups, Math. Annalen 176, 324-333.

P. S. Venkatesan (1972), 'Bisimple left inverse semigroups', Semigroup Forum 4, 34-35.

R. J. Warne (1980), $\mathscr{L}$-Unipotent semigroups', Nigerian J. Sci., to appear.

Miyuki Yamada (1967), 'Regular semigroups whose idempotents satisfy permutation identities', Pac. J. Math. 21, 371-392.

\section{Department of Mathematics}

University College

Trivandrum-695001

Kerala State

India 